\title{
Chromosome Numbers in the Triatominae (Hemiptera-Reduviidae): a Review
}

\section{Francisco Panzera/ ${ }^{+}$, Ruben Pérez*, Sonia Hornos*, Yanina Panzera, Rosario Cestau*, Verónica Delgado, Paula Nicolini}

\author{
Sección Genética Evolutiva, Facultad de Ciencias, Universidad de la República, Tristán Narvaja 1674, 11200 \\ Montevideo, Uruguay *Departamento de Genética, Facultad de Medicina, General Flores 2125, 11800 \\ Montevideo, Uruguay
}

The chromosome numbers of 46 out of the 122 currently recognized species of Triatominae (Hemiptera, Reduviidae) are summarized. We present the number of autosomes, the sex mechanism and the first reference for each karyotype.

Key words: Chagas disease - cytogenetics - holocentric chromosomes -Triatominae

The subfamily Triatominae (HemipteraReduviidae) includes 122 species of hematophagous insects and is divided into five tribes and 15 genera. Schofield (1994) lists 118 species of Triatominae, to which should now be added Belminus laportei (Lent et al. 1995), Rhodnius stali (Lent et al. 1993), Triatoma melanosoma (Lent et al. 1994), and T. gomeznunezi (Martínez et al. 1994).

Triatomines present holocentric chromosomes, which do not have a morphologically differentiated centromere. This feature, together with their small size, hindered the progress in studies of their cytogenetics, which, for a long time, were restricted to descriptions of chromosome number and sex mechanism with conventional staining.

In spite of their medical importance as vectors of Chagas disease, chromosomal studies are limited to a small number of species. Previous revisions reported the chromosome complements (haploid and diploid) of 29 species (Ueshima 1966, 1979). The purpose of this report is to summarize the chromosome numbers of the 46 cytogenetically studied species up to date (Table). Four of them are described for the first time in this paper

${ }^{+}$Corresponding author. E-mail: panzera@ibiol.edu.uy. This research is sponsored by CSIC, CONICYT, PEDECIBA (Uruguay), equipment donation to the Government of Japan and European Communities grants (TS3*CT 91-0029, TS3*CT 92-0092, TS3*CT 920130).

Received 31 October 1995

Accepted 8 February 1996
(Rhodnius pallescens, Triatoma melanosoma, T. picturata and T. tibiamaculata).

Table shows that triatomines have a high chromosomal homogeneity. The most common number of autosomes (A) is 20 , with only three exceptions: Triatoma nitida and Panstrongylus megistus (both with $18 \mathrm{~A}$ ) and T.rubrofasciata (22 A). Three sex mechanisms are found in the males: $X Y(25$ species $), X_{1} X_{2} Y(19$ species $)$ and $\mathrm{X}_{1} \mathrm{X}_{2} \mathrm{X}_{3} \mathrm{Y}(2$ species). In order to verify these multiple sex mechanisms it would be necessary to confirm them in females.

At present, species differentiation (Pérez et al. 1992), intraspecific variations (Panzera et al. 1992) and evolutionary relationships (Panzera et al. 1995) can all be better studied with the aid of banding techniques and the detailed analysis of meiotic chromosome behaviour. This cytogenetic approach is a useful tool for clarifying taxonomic uncertainties, particularly in those groups of epidemiological importance. These studies may allow the differentiation of morphologically similar species such as those belonging to the phyllosoma complex and sordida group. On the other hand, different populations within a given species can be identified. This could be the case for $T$. brasiliensis and $T$. infestans from Bolivia, where sylvatic and domestic populations may be distinguished on the basis of their cytogenetic differences.

\section{ACKNOWLEDGEMENTS}

To Dr J Jurberg (Laboratório Nacional e Internacional de Referência em Taxonomia de Triatomíneos, Instituto Oswaldo Cruz, Rio de Janeiro, Brasil) for providing many species studied here. To Dr C Schofield and JP Dujardin for their valuable comments on the manuscript. 


\section{TABLE}

Chromosome numbers reported in the subfamily Triatominae

\begin{tabular}{|c|c|c|}
\hline Species & $2 n$ & First reference \\
\hline \multicolumn{3}{|l|}{ TRIBU: RHODNIINI } \\
\hline Psammolestes coreodes & $22 \sigma^{\prime \prime}=20 \mathrm{~A}+\mathrm{XY}$ & Schreiber \& Pellegrino 1950 \\
\hline Rhodnius ecuadoriensis & $22 \sigma^{\prime \prime}=20 \mathrm{~A}+\mathrm{XY}$ & Scvortzoff et al. 1996 \\
\hline$R$. nasutus & $22 \sigma^{\prime \prime}=20 A+X Y$ & Pérez et al. 1992 \\
\hline \multirow[t]{2}{*}{ R. neglectus } & $22 \sigma=20 \mathrm{~A}+\mathrm{XY}$ & Barth 1956 \\
\hline & $22 q=20 A+X X$ & Koshy 1979a \\
\hline \multirow[t]{2}{*}{ R. neivai } & $22 \sigma^{\prime \prime}=20 \mathrm{~A}+\mathrm{XY}$ & Koshy $1979 b$ \\
\hline & $22 Q=20 A+X X$ & Koshy $1979 a$ \\
\hline$R$. pallescens & $22 \sigma=20 A+X Y$ & This report \\
\hline \multirow[t]{2}{*}{$R$. pictipes } & $22 \sigma^{\circ}=20 \mathrm{~A}+\mathrm{XY}$ & Pérez et al. 1992 \\
\hline & $22 Q=20 A+X X$ & Koshy $1979 a$ \\
\hline \multirow[t]{2}{*}{$R$. prolixus } & $22 \sigma^{\prime \prime}=20 \mathrm{~A}+\mathrm{XY}$ & Schreiber \& Pellegrino 1950 \\
\hline & $22 O=20 \mathrm{~A}+\mathrm{XX}$ & Koshy $1979 a$ \\
\hline \multirow[t]{2}{*}{ R. robustus } & $22 \sigma^{\prime \prime}=20 \mathrm{~A}+\mathrm{XY}$ & Koshy $1979 b$ \\
\hline & $22 @=20 \mathrm{~A}+\mathrm{XX}$ & Koshy 1979a \\
\hline \multicolumn{3}{|l|}{ TRIBU: TRIATOMINI } \\
\hline Dipetalogaster maximus & $22 \sigma^{\prime \prime}=20 \mathrm{~A}+\mathrm{XY}$ & Ueshima 1966 \\
\hline \multirow[t]{2}{*}{ Mepraia spinolai } & $23 \sigma^{\prime \prime}=20 \mathrm{~A}+\mathrm{X}_{1} \mathrm{X}_{2} \mathrm{Y}$ & Schofield et al. 1996 \\
\hline & $24 Q=20 A+X_{1} X_{1} X_{2} X_{2}$ & Schofield et al. 1996 \\
\hline Panstrongylus herreri & $23 \sigma^{\prime \prime}=20 A+X_{1}^{1} X_{2}^{1} Y^{2}$ & Ueshima 1966 \\
\hline P. megistus & $21 \sigma^{\prime \prime}=18 \mathrm{~A}+\mathrm{X}_{1} \mathrm{X}_{2}^{2} \mathrm{Y}$ & Schreiber \& Pellegrino 1950 \\
\hline P. tupynambai & $23 \sigma^{\prime \prime}=20 \mathrm{~A}+\mathrm{X}_{1}^{1} \mathrm{X}_{2}^{2} \mathrm{Y}$ & Panzera et al. 1991 \\
\hline \multirow{2}{*}{ Paratriatoma hirsuta } & $22 \sigma^{\prime \prime}=20 \mathrm{~A}+\mathrm{XY} \mathrm{Y}^{2}$ & Ueshima 1966 \\
\hline & $22 @=20 A+X X$ & Ueshima 1966 \\
\hline \multirow[t]{2}{*}{ Triatoma barberi } & $23 \sigma^{\prime \prime}=20 A+X_{1} X_{2} Y$ & Ueshima 1966 \\
\hline & $24 Q=20 A+X_{1} X_{1} X_{2} X_{2}$ & Ueshima 1966 \\
\hline \multirow[t]{2}{*}{ T. brasiliensis } & $22 \sigma^{\prime \prime}=20 A+X Y$ & Schreiber \& Pellegrino 1950 \\
\hline & $22 Q=20 A+X X$ & Schreiber et al. 1967 \\
\hline T. circummaculata & $22 \sigma=20 \mathrm{~A}+\mathrm{XY}$ & Scvortzoff et al. 1996 \\
\hline \multirow[t]{2}{*}{ T. delpontei } & $22 \sigma^{\prime \prime}=20 \mathrm{~A}+\mathrm{XY}$ & Ueshima 1966 \\
\hline & $22 \bigcirc=20 A+X X$ & Panzera et al. 1995 \\
\hline \multirow[t]{2}{*}{ T. dimidiata ${ }^{a}$} & $22 \sigma^{\prime \prime}=20 \mathrm{~A}+\mathrm{XY}$ & Schreiber \& Pellegrino 1950 \\
\hline & $23 \sigma=20 \mathrm{~A}+\mathrm{X}_{1} \mathrm{X}_{2} \mathrm{Y}$ & Panzera et al. 1994 \\
\hline T. eratyrusiformis & $24 \sigma=20 \mathrm{~A}+\mathrm{X}_{1}^{1} \mathrm{X}_{2}^{2} \mathrm{X}_{3} \mathrm{Y}$ & Ueshima 1966 \\
\hline T. gerstaeckeri & $23 \sigma=20 \mathrm{~A}+\mathrm{X}_{1} \mathrm{X}_{2} \mathrm{Y}^{\prime}$ & Ueshima 1966 \\
\hline T. guasayana & $22 \sigma^{\prime \prime}=20 \mathrm{~A}+\mathrm{XY}$ & Rebagliati et al. 1995 \\
\hline \multirow{2}{*}{ T. infestans } & $22 \sigma^{\prime \prime}=20 \mathrm{~A}+\mathrm{XY}$ & Schreiber \& Pellegrino 1950 \\
\hline & $22 Q=20 A+X X$ & Schreiber et al. 1967 \\
\hline \multirow[t]{2}{*}{ T. lecticularia } & $22 \sigma^{\prime \prime}=20 A+X Y$ & Ueshima 1966 \\
\hline & $22 Q=20 A+X X$ & Ueshima 1966 \\
\hline T. longipennis & $23 \sigma^{\prime \prime}=20 A+X_{1} X_{2} Y$ & Panzera et al. 1994 \\
\hline \multirow[t]{2}{*}{ T. maculata } & $22 \sigma^{\prime \prime}=20 \mathrm{~A}+\mathrm{XY}$ & Schreiber \& Pellegrino 1950 \\
\hline & $22 ᄋ=20 \mathrm{~A}+\mathrm{XX}$ & Schreiber et al. 1967 \\
\hline T. matogrossensis & $22 \sigma^{\prime \prime}=20 \mathrm{~A}+\mathrm{XY}$ & Pérez et al. 1992 \\
\hline T. mazzottii & $23 \sigma=20 A+X_{1} X_{2} Y$ & Panzera et al. 1994 \\
\hline \multirow{2}{*}{ T. melanosoma } & $22 \sigma^{\prime}=20 \mathrm{~A}+\mathrm{XY}^{1}$ & This report \\
\hline & $22 Q=20 A+X X$ & This report \\
\hline T. nitida & $21 \sigma^{\prime \prime}=18 \mathrm{~A}+\mathrm{X}_{1} \mathrm{X}_{2} \mathrm{Y}$ & Schreiber \& Pellegrino 1950 \\
\hline \multirow{3}{*}{$\begin{array}{l}\text { T. patagonica } \\
\text { T. pallidipennis }\end{array}$} & $22 \sigma^{\prime \prime}=20 \mathrm{~A}+\mathrm{XY}{ }^{2}$ & Ueshima 1966 \\
\hline & $23 \sigma^{\prime \prime}=20 \mathrm{~A}+\mathrm{X}_{1} \mathrm{X}_{2} \mathrm{Y}$ & Ueshima 1966, Panzera et al. 1994 \\
\hline & $22 \sigma^{\prime \prime}=20 A+X Y$ & Koshy 1979 c \\
\hline T. peninsularis & $23 \sigma=20 A+X_{1} X_{2} Y$ & Ueshima 1966 \\
\hline & $24 Q=20 A+X_{1} X_{1} X_{2} X_{2}$ & Ueshima 1966 \\
\hline T. picturata & $23 \sigma^{\prime \prime}=20 \mathrm{~A}+\mathrm{X}_{1} \mathrm{X}_{2} \mathrm{Y}$ & This report \\
\hline T. platensis & $22 \sigma^{\prime \prime}=20 \mathrm{~A}+\mathrm{XY} \mathrm{Y}^{2}$ & Schreiber \& Pellegrino 1950 \\
\hline & $22 Q=20 A+X X$ & Panzera et al. 1995 \\
\hline T. protracta & $23 \sigma^{\prime \prime}=20 A+X_{1} X_{2} Y$ & Ueshima 1966 \\
\hline & $24 Q=20 A+X_{1} X_{1} X_{2} X_{2}$ & Ueshima 1966 \\
\hline
\end{tabular}



T. pseudomaculata
T. rubida
T. rubrofasciata
T. rubrovaria
T. sanguisuga
T. sinaloensis
T. sordida
T. tibiamaculata
T. vitticeps

$$
\begin{aligned}
& 22 \sigma^{\prime \prime}=20 \mathrm{~A}+\mathrm{XY} \\
& 23 \sigma^{\circ}=20 \mathrm{~A}+\mathrm{X}_{1} \mathrm{X}_{2} \mathrm{Y}
\end{aligned}
$$

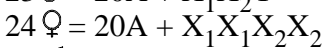

$$
\begin{aligned}
& 25 \sigma=22 \mathrm{~A}+\mathrm{X}_{1} \mathrm{X}_{2} \mathrm{Y}^{2} \\
& 22 \sigma^{\circ}=20 \mathrm{~A}+\mathrm{XY} \\
& 22 \mathrm{O}=20 \mathrm{~A}+\mathrm{XX} \\
& 23 \sigma^{\prime \prime}=20 \mathrm{~A}+\mathrm{X}_{1} \mathrm{X}_{2} \mathrm{Y} \\
& 23 \sigma^{\prime \prime}=20 \mathrm{~A}+\mathrm{X}_{1} \mathrm{X}_{2} \mathrm{Y} \\
& 24 \stackrel{Q}{Q}=20 \mathrm{~A}+\mathrm{X}_{1} \mathrm{X}_{1} \mathrm{X}_{2} \mathrm{X}_{2} \\
& 22 \sigma^{\prime \prime}=20 \mathrm{~A}+\mathrm{XY} \\
& 22 \bigcirc=20 \mathrm{~A}+\mathrm{XX} \\
& 23 \sigma=20 \mathrm{~A}+\mathrm{X}_{1} \mathrm{X}_{2} \mathrm{Y} \\
& 24 \bigcirc=20 \mathrm{~A}+\mathrm{X}_{1} \mathrm{X}_{1} \mathrm{X}_{2} \mathrm{X}_{2} \\
& 24 \sigma^{\circ}=20 \mathrm{~A}+\mathrm{X}_{1} \mathrm{X}_{2} \mathrm{X}_{3} \mathrm{Y}^{2} \\
& 26 \wp=20 A+X_{1} X_{1} X_{2} X_{2} X_{3} X_{3}
\end{aligned}
$$

Schreiber et al. 1972

Ueshima 1966

Ueshima 1966

Manna 1950

Schreiber \& Pellegrino 1950

Scvortzoff et al. 1996

Payne 1909

Ueshima 1966

Ueshima 1966

Schreiber \& Pellegrino 1950

Schreiber et al. 1967

This report

This report

Schreiber \& Pellegrino 1950

Schreiber \& Pellegrino 1950

A: autosomes

$a$ : two different sex mechanisms are reported for the same species. Analysis of individuals (males and females) from natural populations over the whole distribution range is necessary to clarify chromosomal data.

\section{REFERENCES}

Barth R 1956. Estudos anatômicos e histológicos sôbre a subfamília Triatominae (Heteroptera, Reduviidae). VI parte: Estudo comparativo sôbre a espermiocitogênese das espécies mais importantes. Mem Inst Oswaldo Cruz 54: 599-616.

Koshy TK 1979a. Chromosomes of Triatominae I: Haploid Karyotypes of three species in the genus Rhodnius (Hemiptera: Reduviidae). Acta Cient Venezolana 30: 183-190.

Koshy TK 1979b. Chromosomes of Triatominae II: Karyotypes studies of five species in the genus Rhodnius (Hemiptera: Reduviidae). Acta Cient Venezolana 30: 191-195.

Koshy TK 1979c. Chromosomes of Triatominae III: Karyotypes studies of Triatoma pallidipennis (Hemiptera:Reduvidae). Acta Cient Venezolana 30: 196-198.

Lent H, Jurberg J, Galvão C 1993. Rhodnius stali n. sp., afim de Rhodnius pictipes Stal, 1872 (Hemiptera, Reduviidae, Triatominae). Mem Inst Oswaldo Cruz 88: 605-614.

Lent H, Jurberg J, Galvão C, Carcavallo RU 1994. Triatoma melanosoma, novo status para Triatoma infestans melanosoma Martínez, Olmeda \& Carcavallo, 1987 (Hemiptera, Reduviidae). Mem Inst Oswaldo Cruz 89: 353-358.

Lent H, Jurberg J, Carcavallo RU 1995. Belminus laportei sp.n. da Região Amazônica (Hemiptera: Reduviidae: Triatominae). Mem Inst Oswaldo Cruz, 90: 33-39.

Manna GK 1950. Multiple sex chromosome mechanism in a reduviid bug Conorhinus rubrofasciata (De Geer). Proc Zool Soc Bengal 3: 155-161.

Martínez A, Carcavallo RU, Jurberg J 1994. Triatoma gomeznunezi a new species of Triatomini from Mexico (Hemiptera, Reduviidae, Triatominae). Entomología y Vectores 1, 15-19.

Panzera F, Alvarez F, Sanchez-Rufas J, Pérez R, Suja JA, Scvortzoff E, Dujardin JP, Estramil E, Salvatella
R 1992. C-heterochromatin polymorphism in holocentric chromosomes of Triatoma infestans (Hemiptera-Reduviidae). Genome 35:1068-1074.

Panzera F, Hornos S, Cestau R, Panzera Y, Pérez R 1994. Comparative cytogenetic analysis of four mexican species of triatomines (Hemiptera-Reduviidae). Abst XI Latinoamer Congr of Genetics, México, 330.

Panzera F, Pérez R, Panzera Y, Alvarez F, Scvortzoff E, Salvatella R 1995. Karyotype evolution in holocentric chromosomes of three related species of triatomines (Hemiptera-Reduviidae). Chromosome Research 3: 143-150.

Panzera Y, Panzera F, Salvatella R 1991. Análisis cromosómico de Panstrongylus tupynambai Lent 1942 (Hemiptera-Reduviidae). Abst. X Latinoamer. Congr. Parasitology, Uruguay, p. 206.

Payne F 1909. Some new types of chromosome distribution and their relation to sex. Biol Bull 16: 119166.

Pérez R, Panzera Y, Scafiezzo S, Mazzella MC, Panzera F, Dujardin JP, Scvortzoff E 1992. Cytogenetics as a tool for triatomine species distinction (HemipteraReduviidae). Mem Inst Oswaldo Cruz 87: 353-361.

Rebagliati P, Mola L, Papeschi A, Wisnivesky-Colli C 1995. Similitud citogenética entre Triatoma sordida y T. guasayana. Abst. XXVI Cong. Arg. Genetics, Argentina, p. 233

Schofield CJ 1994. Triatominae. Biología y control. UK: Eurocomunica Publications, 77 pp.

Schofield CJ, Apt W, Sagua H, Panzera F, Dujardin JP 1996. Alary polymorphism in Triatoma spinolai, and its possible relationship with demographic strategy. Med Vet Entomol submitted.

Schreiber G, Pellegrino J 1950. Eteropicnosi di autosomi come possible meccanismo di speciazione (Ricerche citologiche su alcuni Emitteri neotropici). Sci Genet 3: 215-226.

Schreiber G, Carvalho HC, Espínola HN 1967. Genetics of Triatoma and other vector species, p. 441456. In JW Wright \& R Pal Genetics of Insect vectors of Disease, Elsevier, Amsterdam. 
Schreiber G, Bogliolo AR, Coelho de Pinho A 1972. Cytogenetics of Triatominae: Caryotype, DNA content, nuclear size and heteropycnosis of autosomes. Rev Brasil Biol 32: 255-263.

Scvortzoff E, Panzera F, Pérez R, Alvarez F, Mazzella MC, Panzera Y, Hornos S, Martínez M, Salvatella $\mathrm{R}$ 1996. Citogenética de Triatominos. In R
Carcavallo. Atlas de Triatominos de América. Argentine. In press.

Ueshima N 1966. Cytotaxonomy of the Triatominae (Reduviidae: Hemiptera). Chromosoma 18: 97-122.

Ueshima N 1979. Insecta 6. Hemiptera II. Heteroptera, p. 117. In B John, Animal Cytogenetics. Gebruder Borntraeger, Berlin, Stuttgart. 